\title{
Aeroelastic Analysis of Unrestrained Aircraft Wing with External Stores Under Roll Maneuver
}

\author{
Seyed Ahmad Fazelzadeh \\ Faculty of Mechanical Engineering, Shiraz University, Molla Sadra Ave, Shiraz, Iran. PO Box: 71345
}

Amir Hosein Ghasemi

Mechanical Engineering Department, Islamic Azad University, Shahr-Babak Branch, Daneshgah Ave, ShahrBabak, Iran. PO Box: 18313

\author{
Abbas Mazidi \\ Mechanical Engineering Department, Yazd University, Daneshgah Ave, Yazd, Iran. PO Box: 47948
}

(Received 29 September 2014; accepted: 20 January 2015)

This paper discusses our study on the flutter of an unrestrained aircraft wing carrying a fuselage at its semispan and arbitrary placed external stores under roll maneuver. Maneuver terms are combined in the governing equations which are obtained using the Hamilton's principle. The wing is represented by a classical beam and incorporates bending-torsion flexibility. Theodorsen unsteady aerodynamic pressure loadings are considered to simulate the aeroelastic loads. The Galerkin method is subsequently applied to convert the partial differential equations into a set of ordinary differential equations. Numerical simulations are validated against several previous published results and good agreement is observed. In addition, simulation results are presented to show the effects of the roll angular velocity, fuselage mass, external stores mass, and their locations on the wing flutter of an aircraft in free-flight condition. Parametric studies show that the predicted flutter boundaries are very sensitive to the aircraft rigid body roll angular velocity, fuselage mass and external stores mass and locations.

\section{NOMENCLATURE}

\section{$b$}

$w_{i}$

Wing length wings

Strain energy

Sweep angle

Twist angle

Air density

\section{Wing semi chord}

Distance between the fuselage center of gravity and wing elastic axis

Distance between the store center of gravity and wing elastic axis

Young's modulus

Shear modulus

Wing cross-section moment of inertia

Wing cross-section polar moment of inertia

Wing sectional lift

Mass of the wing per unit length

Aerodynamic moment

Displacement vector of an arbitrary point of

Kinetic energy

Nondimensional flutter speed

Bending displacement

The external mass location in $x, y$ and $z$ directions, respectively

Variational operator

Strain component

Density of the wing

Stress component

$\begin{array}{ll}\omega_{f} & \text { Flutter frequency } \\ \omega_{\theta} & \text { Torsional frequency } \\ \Omega & \text { Roll angular velocity }\end{array}$

\section{INTRODUCTION}

The flutter prediction of an unrestrained aircraft wing with stores is of paramount importance for the analysis and design of an aircraft. Clearly, estimating the aeroelastic instabilities of such aircraft with different wing configurations is critical to establish the flight envelope of newly designed aircrafts.

Many of the previous efforts made to simulate wing flutter have considered uniform straight wings with external stores. One of the first works devoted to the aeroelasticity of aircraft wings with external store is the paper by Goland and Luke on the determination of the flutter speed of a uniform cantilever wing with tip mass. ${ }^{1}$ They verified the flutter speed by integration of the differential equations of the wing motion. Harry and Charles ${ }^{2}$ analyzed the flutter of a uniform wing and made a comparison between the analytical and the experimental results. Lottati considered the aeroelastic stability of a swept wing with tip weights for an unrestrained vehicle. $^{3}$ In his work, a composite wing has been studied, and it was observed that flutter occurs at a lower speed as compared with a clean wing configuration. Gern and Librescu have made some efforts to show the effects of externally mounted masses on the static and dynamic aeroelasticity of advanced swept cantilevered wings. ${ }^{4,5}$ The dynamic response of adaptive cantilevered beams carrying externally mounted stores and exposed to time-dependent external excitations has been con- 
sidered by $\mathrm{Na}$ and Librescu. ${ }^{6}$ Moreover, Librescu and Song ${ }^{7}$ investigated the free vibration and dynamic response to external time-dependent loads of aircraft wings carrying eccentrically located heavy stores. They have modeled the wing as a thin-walled anisotropic composite beam. Also, Edwards and Wieseman ${ }^{8}$ studied the flutter and divergence of three check cases that include unrestrained airfoils and wing models. The bending-torsional flutter characteristics of an aircraft wing containing an arbitrarily placed mass under a follower force have been studied by Fazelzadeh et al. ${ }^{9}$ They showed the important influence of the location and magnitude of the store mass and the follower force on the flutter speed and frequency of the wing. Also, Mazidi and Fazelzadeh ${ }^{10}$ investigated the flutter of an aircraft wing with a powered engine. They confirmed that the engine thrust and locations have a considerable effect on the wing aeroelastic stability region.

Although these works and several others addressed the problem of the wing-store aeroelasticity, the effect of the aircraft maneuvers on the unrestrained aircraft wing instability has not received much attention in the literature and only few works about the maneuver effects on the aeroelastic behavior of cantilever wing-stores configuration have been conducted. ${ }^{11,12}$ The maneuver has a significant influence on the dynamic response and instability of the wing-store configuration, especially for unrestrained aircraft wing. Since rigid body rotations due to maneuver angular velocities, such as the one produced by a roll maneuver, can adversely affect the aircraft aeroelastic stability region, it is critical to include maneuvering angular velocities in aeroelastic analysis.

To add to the aforementioned bulk of literature in this field, the aeroelastic modeling and flutter study of an unrestrained aircraft under roll maneuver is considered in this study. The aircraft is modeled as a wing carrying a fuselage at its semispan and arbitrarily placed external stores.

\section{GOVERNING EQUATIONS}

An unrestrained aircraft with swept wings, shown in Fig. 1, is considered. As it can be seen in Fig. 1(b) the aircraft fuselage is modeled as a concentrated mass and inertia located at the center of the unrestrained swept wings. The structural flexibility as well as the roll angular velocity is taken into account when deriving the aeroelastic governing equations. The wing model is valid for long, straight, homogeneous beams and is derived based on the Goland wing model.

The equations of motion and boundary conditions are derived using Hamilton's variational principle that may be expressed as: ${ }^{13}$

$$
\begin{gathered}
\int_{t_{1}}^{t_{2}}\left[\delta U_{T}-\delta T_{T}-\delta W_{T}\right] d t=0 \\
\delta w=\delta \theta=0 \quad \text { at } \quad t=t_{1}=t_{2}
\end{gathered}
$$

where $U$ and $T$ are strain energy and kinetic energy, and $W$ is the work done by non-conservative forces. Also, the subscript ' $T$ ' means the total system. The use of Hamilton's principle is especially convenient in cases of unusual boundary conditions because the equation(s) of motion and boundary conditions are determined in a unified procedure. Therefore, the Hamilton's

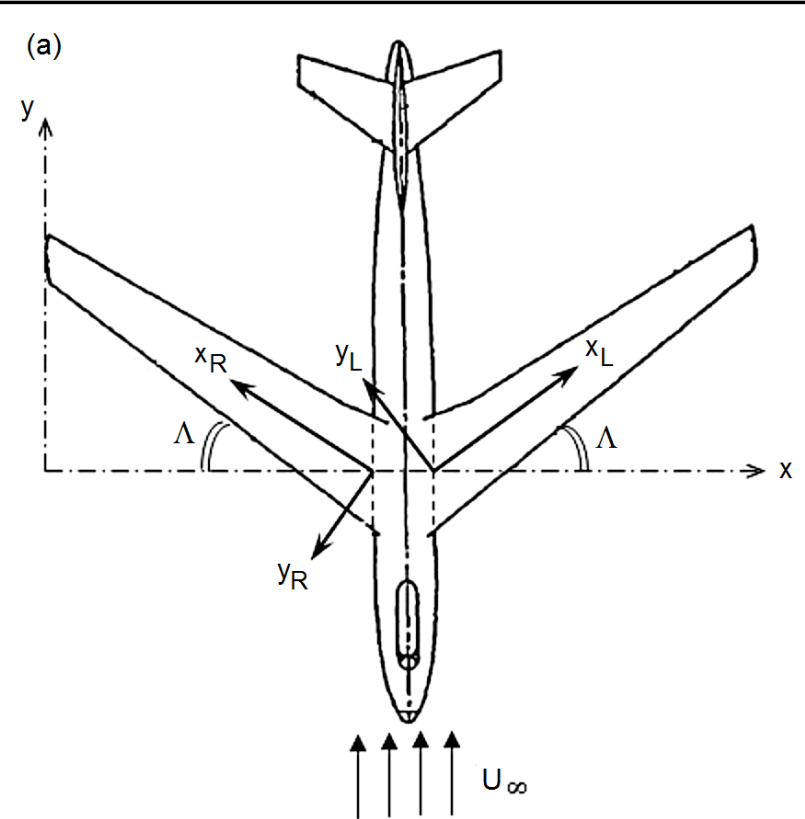

(b)

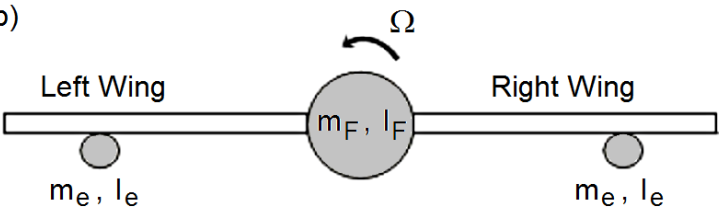

Figure 1. Schematic of an unrestrained aircraft wing under roll maneuver, (a) Top view; (b) Front view.

principle yields the equations of motion in the form of partial differential equations with accompanying boundary conditions.

The kinetic energy can be divided into three parts: wing, fuselage, and store, i.e. $T_{T}=T_{w}+T_{e}+T_{F}$. The subscripts $w, e$, and $F$ identify the wing, externally mounted mass, and aircraft fuselage, respectively. The first variation of the wing kinetic energy is:

$$
\delta T_{w}=\delta T_{w_{1}}+\delta T_{w_{2}}
$$

where $\delta T_{w_{1}}$ and $\delta T_{w_{2}}$ are the first variation of the right and left wing kinetic energy, respectively. Also,

$$
\begin{aligned}
& \delta T_{w_{1}}=\int_{0}^{l} \iint_{A} \rho \dot{\mathbf{R}}_{w_{1}} \delta \dot{\mathbf{R}}_{w_{1}} d x d A \\
& \delta T_{w_{2}}=\int_{0}^{l} \iint_{A} \rho \dot{\mathbf{R}}_{w_{2}} \delta \dot{\mathbf{R}}_{w_{2}} d x d A .
\end{aligned}
$$

$\mathbf{R}_{w}$ 's are displacement vectors of arbitrary points of the right and left wings that are given by

$$
\begin{aligned}
& \mathbf{R}_{w_{1}}=\mathbf{R}_{O_{1}}+\mathbf{r}_{w_{1}}=\left(R_{X} \cos \Lambda-R_{Y} \sin \Lambda\right) \mathbf{i}_{1}+ \\
& \quad\left(R_{X} \sin \Lambda+R_{Y} \cos \Lambda\right) \mathbf{j}_{1}+\left[w_{1}+\left(y_{1}-a b\right) \theta_{1}-R_{Z}\right] \mathbf{k}_{1} ; \\
& \mathbf{R}_{w_{2}}=\mathbf{R}_{O_{2}}+\mathbf{r}_{w_{2}}=\left(R_{X} \cos \Lambda-R_{Y} \sin \Lambda\right) \mathbf{i}_{2}+ \\
& \quad\left(-R_{X} \sin \Lambda-R_{Y} \cos \Lambda\right) \mathbf{j}_{2}+\left[w_{2}+\left(y_{2}+a b\right) \theta_{2}-R_{Z}\right] \mathbf{k}_{2} ;
\end{aligned}
$$

where $\mathbf{R}_{O}$ 's are the wings root position vector with respect to airplane center of gravity, shown in Fig. 1 and $\mathbf{r}_{w}$ 's are the 
position vector of arbitrary points of the wings with respect to the wings root. The velocity vector of any points on the wing can be obtained through transport theorem as below: ${ }^{14}$

$$
\dot{\mathbf{R}}_{w_{i}}=\frac{\partial \mathbf{R}_{w_{i}}}{\partial t}+\left(\boldsymbol{\Omega} \times \mathbf{R}_{w_{i}}\right)+\mathbf{V}_{\text {airplane }} .
$$

In this equation, two first terms represent the velocity of the point and refer to the coordinate system located on the airplane center of gravity. The third term represents the velocity of the airplane and refers to the inertial reference coordinate system located on Earth:

$$
\mathbf{V}_{\text {airplane }}=v_{x a} \hat{\mathbf{I}}+v_{y a} \hat{\mathbf{J}}+v_{z a} \hat{\mathbf{K}} .
$$

Here it is assumed that the airplane has roll maneuver, so the angular velocity equations of the airplane that refer to right and left wing coordinate systems are:

$$
\begin{aligned}
& \boldsymbol{\Omega}_{1}=-\Omega \sin \Lambda \mathbf{i}_{1}+\Omega \cos \Lambda \mathbf{j}_{1} \\
& \boldsymbol{\Omega}_{2}=-\Omega \sin \Lambda \mathbf{i}_{2}+\Omega \cos \Lambda \mathbf{j}_{2} .
\end{aligned}
$$

By substitution of Eqs. (3)-(7) in Eq. (2), the first variation of the wing kinetic energy can be represented. Using the same kinematical procedure, the first variation of the engine kinetic energy can be derived as:

$$
\begin{aligned}
& \delta T_{e}=\sum_{i=1}^{N} m_{e} \delta_{D}\left(x-x_{e i}\right)\left\{\left[-\ddot{w}_{1}-e_{p} \cos \Lambda \ddot{\theta}_{1}-\ddot{H}+R_{X} \alpha+\right.\right. \\
& \left.2 \alpha R_{Y} \sin \Lambda \cos \Lambda+\Omega^{2} w_{1}+\Omega^{2} e_{p} \cos \Lambda \theta_{1}-\Omega^{2} R_{Z}\right] \delta w_{1}+ \\
& {\left[-e_{p} \cos \Lambda \ddot{w}_{1}-k_{e}^{2} \cos ^{2} \Lambda \ddot{\theta}_{1}-e_{p} \cos \Lambda \ddot{H}+\right.} \\
& e_{p} \cos \Lambda R_{X} \alpha+2 \alpha R_{Y} e_{p} \sin \Lambda \cos ^{2} \Lambda+e_{p} \cos \Lambda \Omega^{2} w_{1}+ \\
& \left.\left.k_{e}^{2} \cos ^{2} \Lambda \Omega^{2} \theta_{1}-e_{p} \cos \Lambda \Omega^{2} R_{Z}\right] \delta \theta_{1}\right\}+ \\
& \sum_{i=1}^{N} m_{e} \delta_{D}\left(x-x_{e i}\right)\left\{\left[-\ddot{w}_{2}-e_{p} \cos \Lambda \ddot{\theta}_{2}-\ddot{H}-R_{X} \alpha+\right.\right. \\
& \left.\Omega^{2} w_{2}+\Omega^{2} e_{p} \cos \Lambda \theta_{2}-\Omega^{2} R_{Z}\right] \delta w_{2}+ \\
& {\left[-e_{p} \cos \Lambda \ddot{w}_{2}-k_{e}^{2} \cos ^{2} \Lambda \ddot{\theta}_{2}-e_{p} \cos \Lambda \ddot{H}-\right.} \\
& e_{p} \cos \Lambda R_{X} \alpha+e_{p} \cos \Lambda \Omega^{2} w_{2}+k_{e}^{2} \cos { }^{2} \Lambda \Omega^{2} \theta_{2}- \\
& \left.\left.e_{p} \cos \Lambda \Omega^{2} R_{Z}\right] \delta \theta_{2}\right\} .
\end{aligned}
$$

In this equation, $x_{e}$ denotes the store distance from the wing root, and $e_{p}$ is the distance between the engine center of gravity and the wing elastic axis. Also, $m_{e}$ and $k_{e}$ are the store mass and radius of gyration, respectively. It should be noted that the velocity vector of any point on the engine, as before, is obtained through transport theorem. Also, the first variation of the fuselage kinetic energy can be derived as:

$$
\begin{aligned}
\delta T_{F}= & m_{F} \vec{V}_{F} \delta \vec{V}_{F} \\
= & {\left.\left[-m_{F} \ddot{w}_{1}-e_{F} \ddot{\theta}_{1}-\ddot{H}+\Omega^{2} w_{1}+\Omega^{2} e_{F} \theta_{1}\right] \delta w_{1}\right|_{x=l}+} \\
& \quad\left[-m_{F} e_{F} \ddot{w}_{1}-m_{F} k_{F}^{2} \ddot{\theta}_{1}-m_{F} e_{F} \ddot{H}+m_{F} e_{F} \Omega^{2} w_{1}+\right. \\
& \left.\quad m_{F} k_{F}^{2} \Omega^{2} e_{F} \theta_{1}\right]\left.\delta \theta_{1}\right|_{x=l}
\end{aligned}
$$

where $m_{F}$ and $k_{F}$ are the fuselage mass and radius of gyration, respectively, and $e_{F}$ is the distance between the fuselage center of gravity and the wing elastic axis.
The strain energy is considered next. The total strain energy, normally, consists of wing, fuselage, and store strain energy. Here, it is assumed that the fuselage and stores are rigid bodies. Consequently, the total strain energy is equal to the wing strain energy. The first variation of the strain energy is: ${ }^{14}$

$$
\delta U=\int_{V}\left[\sigma_{x x} \delta \varepsilon_{x x}+\sigma_{x \eta} \delta \varepsilon_{x \eta}+\sigma_{x \xi} \delta \varepsilon_{x \xi}\right] d x d A
$$

where $\sigma_{i j}$ and $\varepsilon_{i j}$ are stress and strain components, respectively. $\eta$ and $\xi$ are the principal axes of the wing cross section that defines a local coordinate system on the shear center of the cross section.

The use of strain-displacement relations, together with the generalized Hooke's law, permits the strain energy to be expressed in terms of deformation quantities. ${ }^{15}$ Using these expressions and integrating by parts, Eq. (10) recasts as:

$$
\begin{gathered}
\delta U_{w_{1}}=\int_{0}^{l}\left\{\left[E I w_{1}^{\prime \prime \prime \prime}+a b E I \theta_{1}^{\prime \prime \prime \prime}\right] \delta w_{1}+\right. \\
\left.\left[a b E I w_{1}^{\prime \prime \prime \prime}+S \theta_{1}^{\prime \prime \prime \prime}-G J \theta_{1}^{\prime \prime}\right] \delta \theta_{1}\right\} d x \\
\delta U_{w_{2}}=\int_{l}^{2 l}\left\{\left[E I w_{2}^{\prime \prime \prime \prime}+a b E I \theta_{2}^{\prime \prime \prime \prime}\right] \delta w_{2}+\right. \\
\left.\left[a b E I w_{2}^{\prime \prime \prime \prime}+S \theta_{2}^{\prime \prime \prime \prime}-G J \theta_{2}^{\prime \prime}\right] \delta \theta_{2}\right\} d x
\end{gathered}
$$

where $\delta U_{w_{1}}$ and $\delta U_{w_{2}}$ are the right and left wing strain energy, respectively.

The virtual work of the aerodynamic forces acting on the wing may be expressed as:

$$
\delta W_{n c}=\int_{0}^{l}\left(L_{1} \delta w_{1}+M_{1} \delta \theta_{1}\right) d x+\int_{l}^{2 l}\left(L_{2} \delta w_{2}+M_{2} \delta \theta_{2}\right) d x
$$

where $L$ and $M$ are aerodynamic lift and moment, respectively. It should be noted here that the engine aerodynamic is not accounted for in governing equations. Aerodynamic lift and moment are derived from Theodorsen's unsteady thin-airfoil theory. These are:

$$
\begin{gathered}
L(x, t)=-\pi \rho_{\infty} \omega^{2} b^{3}\left[\frac{w}{b} L_{h h}+\frac{\partial w}{\partial x} L_{h h^{\prime}}+\theta_{e f f} L_{h \theta}+\right. \\
\left.b \frac{\partial \theta_{e f f}}{\partial x} L_{h \theta^{\prime}}\right] \\
M(x, t)=\pi \rho_{\infty} \omega^{2} b^{4}\left[\frac{w}{b} M_{\theta h}+\frac{\partial w}{\partial x} M_{\theta h^{\prime}}+\theta_{\text {eff }} M_{\theta \theta}+\right. \\
\left.b \frac{\partial \theta_{e f f}}{\partial x} M_{\theta \theta^{\prime}}\right]
\end{gathered}
$$

where $L_{h h}, L_{h h^{\prime}}, \ldots, M_{\theta \theta^{\prime}}$ are the aerodynamic coefficients. ${ }^{16,17}$

By substituting Eqs. (3)-(12) into Eq. (1), and noticing that for every admissible variation $\left(\delta w_{1}, \delta \theta_{1}, \delta w_{2}, \delta \theta_{2}\right)$ the coefficient of these variations must be zero, the aeroelastic governing 
equations are obtained as:

$$
\begin{aligned}
& -E I w_{1}^{\prime \prime \prime \prime}+m \ddot{w}_{1}+m x_{\theta} \ddot{\theta}_{1}+m \ddot{H}-m R_{X} \alpha- \\
& 2 m R_{Y} \alpha \sin \Lambda \cos \Lambda-m \Omega^{2} w_{1}-m x_{\theta} \Omega^{2} \theta_{1}+m \Omega^{2} R_{Z}+ \\
& \left.m_{F}\left(\ddot{w}_{1}+e_{F} \ddot{\theta}_{1}+\ddot{H}-\Omega^{2} w_{1}-\Omega^{2} e_{F} \theta_{1}\right)\right|_{x=l}+ \\
& \sum_{i=1}^{N} m_{e} \delta_{D}\left(x_{1}-x_{e i}\right)\left(\ddot{w}_{1}+e_{p} \cos \Lambda \ddot{\theta}_{1}+\ddot{H}-R_{X} \alpha-\right. \\
& \left.\quad 2 R_{Y} \alpha \sin \Lambda \cos \Lambda-\Omega^{2} w_{1}-\Omega^{2} e_{p} \cos \Lambda \theta_{1}+\Omega^{2} R_{Z}\right)- \\
& \quad L_{1}=0
\end{aligned}
$$

$$
\begin{aligned}
& -G J \theta_{1}^{\prime \prime}+m x_{\theta} \ddot{w}_{1}+I_{\theta} \ddot{\theta}_{1}+m x_{\theta} \ddot{H}-m x_{\theta} R_{X} \alpha- \\
& 2 m x_{\theta} R_{Y} \alpha \sin \Lambda \cos \Lambda-m x_{\theta} \Omega^{2} w_{1}-I_{\theta} \Omega^{2} \theta_{1}+m x_{\theta} \Omega^{2} R_{Z}+ \\
& \sum_{i=1}^{N} m_{e} \delta_{D}\left(x_{1}-x_{e i}\right)\left(e_{p} \cos \Lambda \ddot{w}_{1}+k_{e}^{2} \cos ^{2} \Lambda \ddot{\theta}_{1}+e_{p} \cos \Lambda \ddot{H}-\right. \\
& \quad e_{p} \cos \Lambda R_{X} \alpha-2 e_{p} R_{Y} \alpha \sin \Lambda \cos ^{2} \Lambda-e_{p} \cos \Lambda \Omega^{2} w_{1}- \\
& \left.k_{e}^{2} \cos ^{2} \Lambda \Omega^{2} \theta_{1}+e_{p} \cos \Lambda \Omega^{2} R_{Z}\right)-M_{1}=0 ;
\end{aligned}
$$

$$
\begin{aligned}
& -E I w_{2}^{\prime \prime \prime \prime}+m \ddot{w}_{2}+m x_{\theta} \ddot{\theta}_{2}+m \ddot{H}-m R_{X} \alpha- \\
& m \Omega^{2} w_{2}-m x_{\theta} \Omega^{2} \theta_{2}+m \Omega^{2} R_{Z}+ \\
& \sum_{i=1}^{N} m_{e} \delta_{D}\left(x_{2}-x_{e i}\right)\left(\ddot{w}_{2}+e_{p} \cos \Lambda \ddot{\theta}_{2}+\ddot{H}+R_{X} \alpha-\Omega^{2} w_{2}-\right. \\
& \left.\quad \Omega^{2} e_{p} \cos \Lambda \theta_{2}+\Omega^{2} R_{Z}\right)-L_{2}=0
\end{aligned}
$$

$$
\begin{aligned}
& -G J \theta_{2}^{\prime \prime}+m x_{\theta} \ddot{w}_{2}+I_{\theta} \ddot{\theta}_{2}+m x_{\theta} \ddot{H}-m x_{\theta} R_{X} \alpha- \\
& m x_{\theta} \Omega^{2} w_{2}-I_{\theta} \Omega^{2} \theta_{2}+m x_{\theta} \Omega^{2} R_{Z}+ \\
& \sum_{i=1}^{N} m_{e} \delta_{D}\left(x_{2}-x_{e i}\right)\left(e_{p} \cos \Lambda \ddot{w}_{2}+k_{e}^{2} \cos ^{2} \Lambda \ddot{\theta}_{2}+e_{p} \cos \Lambda \ddot{H}+\right. \\
& \quad e_{p} \cos \Lambda R_{X} \alpha-e_{p} \cos \Lambda \Omega^{2} w_{2}-k_{e}^{2} \cos ^{2} \Lambda \Omega^{2} \theta_{2}+ \\
& \left.\quad e_{p} \cos \Lambda \Omega^{2} R_{Z}\right)-M_{2}=0 .
\end{aligned}
$$

\section{SOLUTION METHODOLOGY}

Due to intricacy of aeroelastic governing equations, it is difficult to get the exact solution. Therefore, in order to solve the aeroelastic governing equations in a general way, the Galerkin's method is used. ${ }^{18}$ To this end, $w_{i}, \theta_{i}$ (bending and torsion generalized coordinates) are represented by means of series of trial functions, $\varphi_{j_{i}}$, that should satisfy the boundary conditions, multiplied by time dependent generalized coordinates, $\mathbf{q}_{j_{i}}$ :

$$
w_{i}=\varphi_{1_{i}}^{T} \mathbf{q}_{1_{i}} ; \quad \theta_{i}=\varphi_{2_{i}}^{T} \mathbf{q}_{2_{i}} .
$$

The following family of orthogonal functions for $w$ and $\theta$ is used here: ${ }^{16}$

$$
\begin{gathered}
\varphi_{1_{i}}=\frac{(x / l)^{1+i}\left\{6+i^{2}(1-x / l)^{2}+i\left[5-6 x / l+(x / l)^{2}\right]\right\}}{i(1+i)(2+i)(3+i)} \\
\varphi_{2_{i}}=\sin \left(\frac{i \pi x}{l}\right) .
\end{gathered}
$$

Table 1. Validation of the flutter speed and frequency for a wing in free flight condition.

\begin{tabular}{||l|c|c|c|c||}
\hline Method & $\begin{array}{c}\text { Flutter } \\
\text { Speed } \\
(\mathrm{m} / \mathrm{s})\end{array}$ & $\begin{array}{c}\text { Error } \\
(\%)\end{array}$ & $\begin{array}{c}\text { Flutter } \\
\text { Frequency } \\
(\mathrm{rad} / \mathrm{s})\end{array}$ & $\begin{array}{c}\text { Error } \\
(\%)\end{array}$ \\
\hline Exact (Goland \& Luke $\left.^{1}\right)$ & 292.72 & - & 19.17 & - \\
Lottati $^{3}$ & 289.68 & 1.04 & 19.42 & 1.3 \\
Gern \& Librescu $^{5}$ & 293.61 & 0.31 & 21.68 & 13.09 \\
Present & 308.72 & 5.46 & 18.30 & 4.53 \\
\hline
\end{tabular}

Two bending modes and two torsion modes are considered for each wing. By applying the Galerkin procedure on governing equations and using orthogonal properties in the required integrations, the following set of ordinary differential equations are obtained:

$$
\mathbf{M} \ddot{\mathbf{q}}+\mathbf{C} \dot{\mathbf{q}}+\mathbf{K q}=0
$$

Herein, $\mathbf{M}, \mathbf{C}$, and $\mathbf{K}$ denote the mass matrix, the damping matrix and the stiffness matrix, respectively, while $\mathbf{q}$ is the overall vector of generalized coordinates. Finally, Eq. (21) converts to

$$
\dot{\mathbf{Z}}=[\mathbf{A}] \mathbf{Z}
$$

where the state vector $\mathbf{Z}$ is defined as

$$
\mathbf{Z}=\left\{\begin{array}{ll}
\mathbf{q}^{T} & \dot{\mathbf{q}}^{T}
\end{array}\right\}^{T}
$$

and the system matrix $[\mathbf{A}]$ has the form

$$
[\mathbf{A}]=\left[\begin{array}{cc}
{[\mathbf{0}]} & {[\mathbf{I}]} \\
-[\mathbf{M}]^{-1}[\mathbf{K}] & -[\mathbf{M}]^{-1}[\mathbf{C}]
\end{array}\right] .
$$

The problem is now reduced to that of finding out the eigenvalues of matrix $[\mathbf{A}]$ for given values of the air speed parameter $U_{\infty}$. The eigenvalue $\omega$ is a continuous function of the air speed $U_{\infty}$. For $U_{\infty} \neq 0, \omega$ is in general complex, $\omega=\operatorname{Re}(\omega)+i \operatorname{Im}(\omega)$. When $\operatorname{Re}(\omega)=0$ and $\operatorname{Im}(\omega) \neq 0$, the wing is said to be in critical flutter condition. At some point, as $U_{\infty}$ increases, $\operatorname{Re}(\omega)$ turns from negative to positive so that the motion turns from asymptotically stable to unstable.

\section{RESULTS}

As stated in the previous section, the solution to this aeroelastic problem through the extended Galerkin method is sought by using a numerical integration scheme. Two bending modes and two torsion modes for each wing are considered in the solution procedure to this end. The effects of the external mass value and location and the roll angular velocity on the flutter speed of unrestrained wings are simulated. Relevant data for the particular wing-weight combination used here are the same as those utilized in the work of Goland and Luke. ${ }^{1}$ Also, dimensionless parameters used in the numerical simulation are

$$
v_{f}=\frac{U_{f}}{b \omega_{\theta}} ; \quad \omega_{f}=\frac{\bar{\omega}_{f}}{\omega_{\theta}} ; \quad X_{e}=\frac{x_{e}}{l} ; \quad \eta_{F}=\frac{m_{F}}{m l} ; \quad \eta_{e}=\frac{m_{e}}{m l} ;
$$

where $U_{f}$ and $\bar{\omega}_{f}$ are the flutter speed and frequency, respectively.

Our results, without the angular roll velocity, are in good agreement and have been obtained with results from previously published papers, as shown in Table 1. 


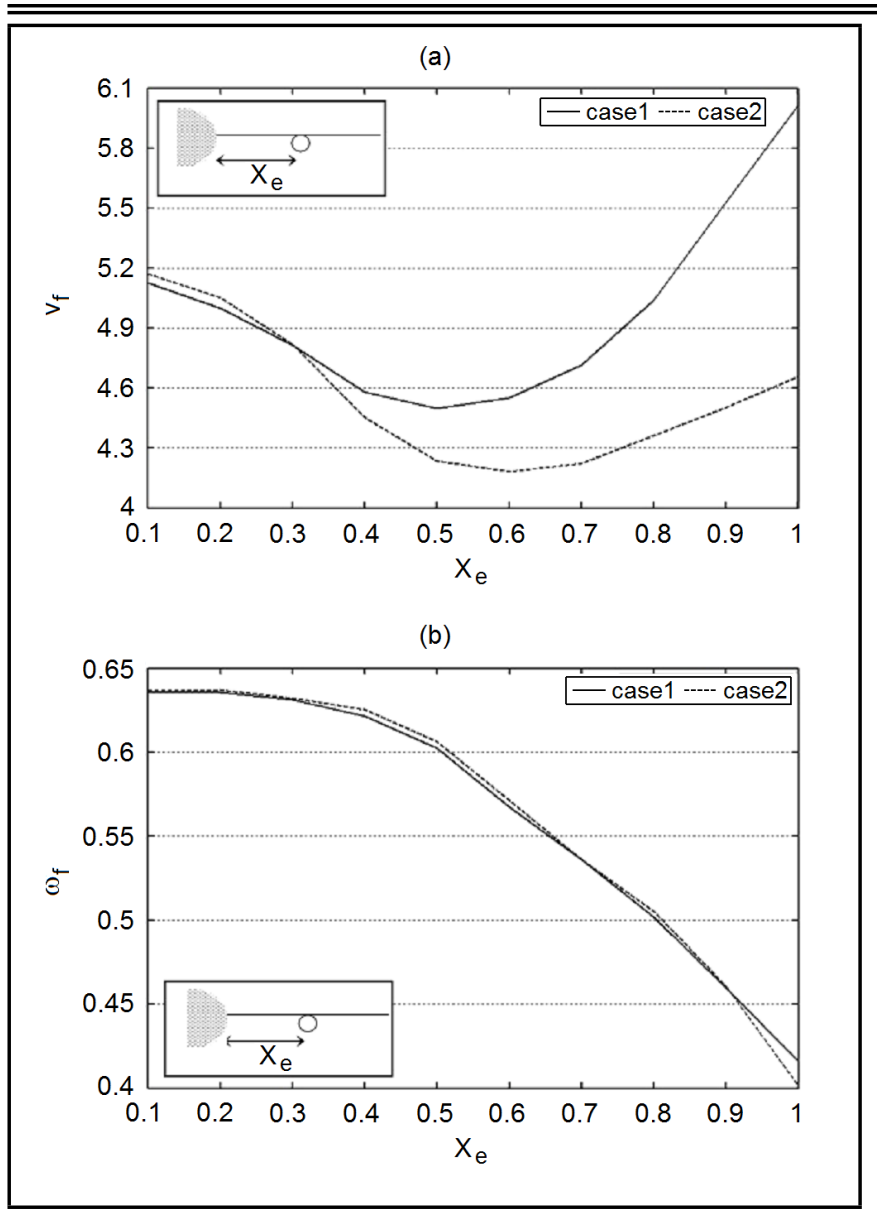

Figure 2. Effects of the spanwise position of the external mass on the aircraft flutter boundary for case1 $\left(e_{p}=0\right)$ and case2 ( $\left.e_{p}=0.63\right)$. (a) Flutter speed; (b) Flutter frequencies.

\subsection{Non-Maneuver Aircraft}

First, the flutter results of the non-maneuvering unrestrained aircraft are considered. Figure 2 shows a parametric study investigating the effect of the store spanwise location on the flutter speed and frequency for two selected values of $e_{p}$. The store mass is assumed to be $\eta_{e}=0.5$, and the fuselage mass is $\eta_{F}=0.5$. The lowest value of the flutter speed takes place around $X_{e}=0.6$. So, one can say that this point is the critical location for store mounting for this wing characteristic.

This behavior is the same as those reported in previous published papers. ${ }^{5,10}$ Also, it can be seen in this figure that increasing the distance of the engine center of gravity from the wing elastic axis will decrease the flutter speed. Figure 2(b) also reveals that the flutter frequency drops in the usual way by moving the external mass towards the wing tip.

The effect of the store mass on the flutter boundary and corresponding flutter frequency of the unrestrained wing is illustrated in Fig. 3 for two selected values of $e_{p}$. The store is assumed to be placed at the middle of the wing span and the fuselage mass is $\eta_{F}=0.5$. Figure 3(a) shows that increasing the store mass restricts the stability region. Also, effects of the store distance from the wing elastic axis on the wing flutter are clear in this figure. Figure 3(b) illustrates that increasing the engine mass value will decrease the flutter frequency, noticeably.

Figure 4 shows the variation of the flutter speed and frequency of the wing due to variations in the aircraft fuselage

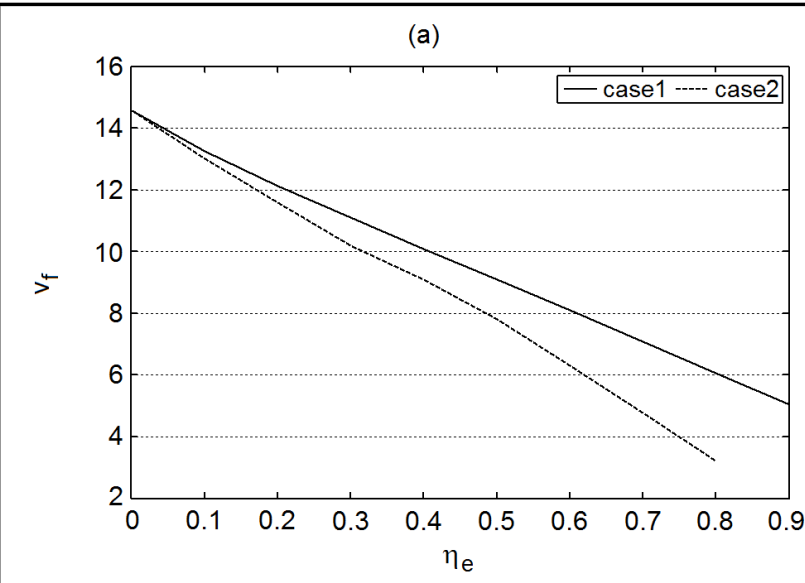

(b)

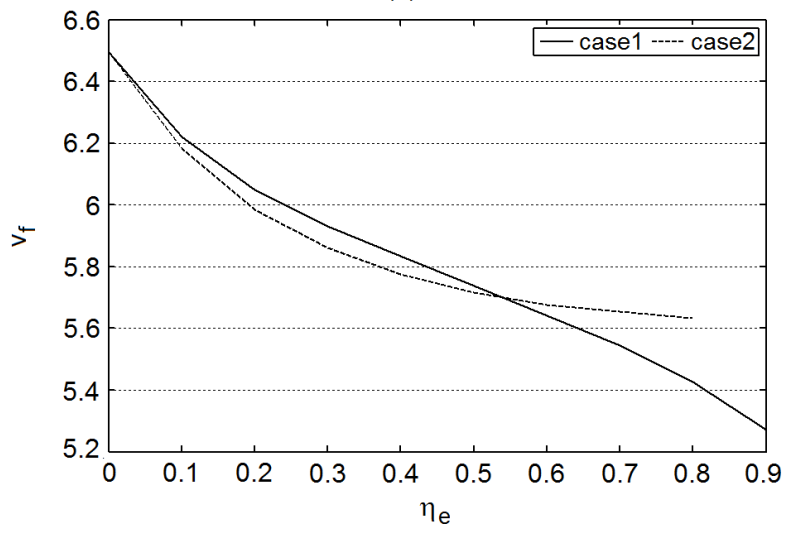

Figure 3. Effects of the store mass on the aircraft flutter boundary for case 1 $\left(e_{p}=0\right)$ and case $2\left(e_{p}=0.63\right)$. (a) Flutter speed; (b) Flutter frequencies.

mass for selected values of $e_{p}$. The results show that an increase of fuselage mass can induce a higher flutter speed. Also, the effect of the store distance from the wing elastic axis on the flutter speed and frequency is clearly highlighted.

\subsection{Rolling Maneuver Aircraft}

This subsection focuses on the flutter simulations of the unrestrained aircraft under roll maneuver. The effect of the store mass on the flutter boundary and corresponding flutter frequency is illustrated in Fig. 5 for different values of the roll angular velocity.

The stores are assumed to be placed at the middle of the wings span and the nondimensional fuselage mass is $\eta_{F}=1$. Figure 5(a) shows that the stability region is limited when the larger external mass is attached to the wing. This is almost independent of the roll angular velocity. Also, effects of the roll maneuver angular velocity on postponing the wing flutter are clear in this figure. Figure 5(b) illustrates that increasing the store mass value will decrease the flutter frequency, noticeably, for all values of the aircraft roll angular velocity.

The influence of the spanwise location of the store on the flutter speed and frequency of the unrestrained wing for selected values of the roll angular velocity is shown in Fig. 6. In this case, the store and fuselage mass ratio is $\eta_{e}=\eta_{F}=1$ and the store is assumed to be located at the wing elastic axis. It can be seen in Fig. 6(a) that increasing the distance of the stores from the wing root, in this case, increases the flutter speed. Also, it can be seen that increasing the roll angular ve- 


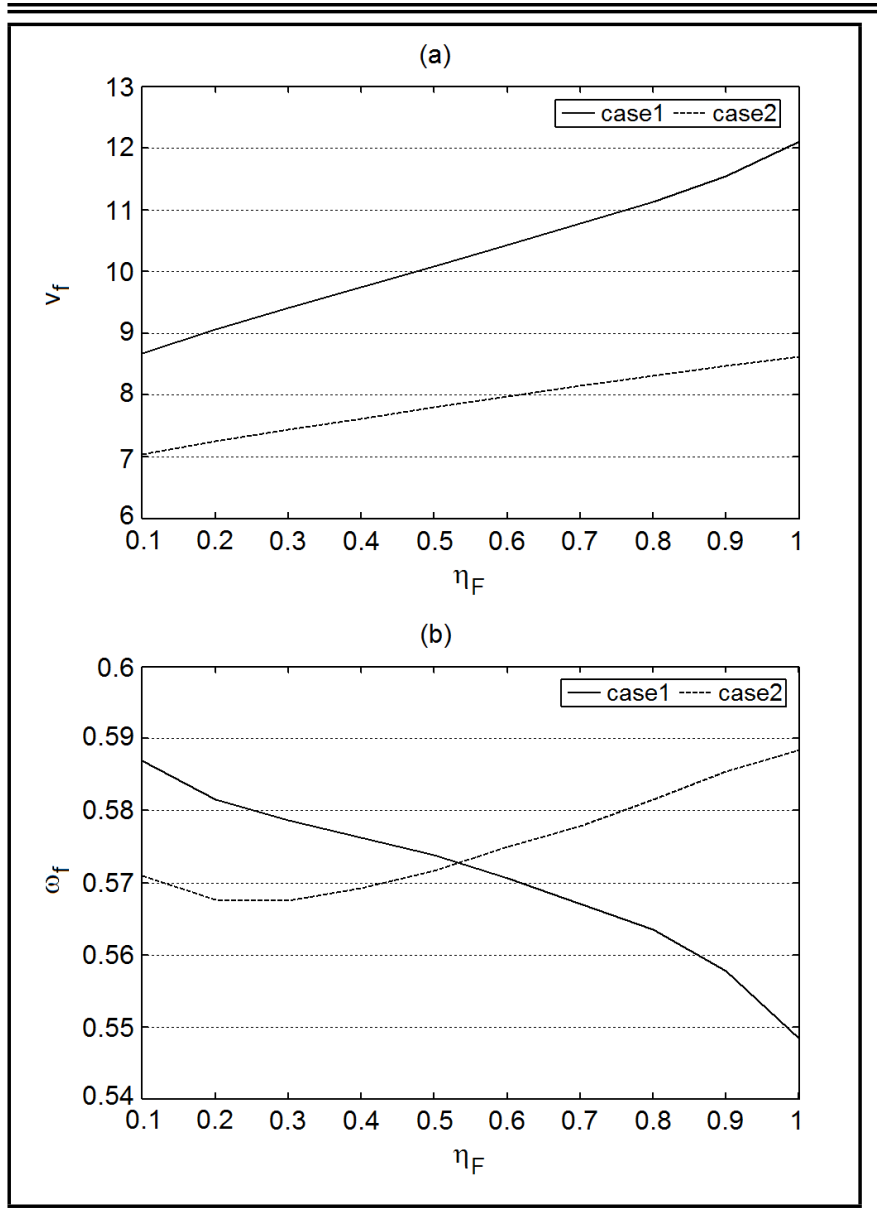

Figure 4. Effects of the fuselage mass on the aircraft flutter boundary for case 1 $\left(e_{p}=0\right)$ and case2 ( $\left.e_{p}=0.63\right)$. (a) Flutter speed; (b) Flutter frequencies.

locity will decrease the flutter speed, noticeably. Figure 6(b) also reveals that the flutter frequency drops in the usual way by moving the stores towards the wings tip. Moreover, the magnitude of the roll angular velocity has noticeable influence on the flutter frequency.

Figure 7 demonstrates the effect of the spanwise location of the stores on the flutter speed and frequency of the unrestrained aircraft with four stores for the selected values of the roll angular velocities. For every wing, the first store is assumed to be placed at the $X_{e_{1}}=0.3$, and only the second store slides from the middle of the wing to the wing tip. Both stores and fuselage have equal mass ratio of $\eta_{e_{1}}=\eta_{e_{2}}=\eta_{F}=1$. It is clear from Fig. 6(a) that increasing the distance of the second engine from the wing root, in this case, will increase the flutter speed. Influence of the second engine spanwise location on the flutter frequency is shown in Fig. 6(b). It can be seen that sliding the engine toward the wing tip will decrease the flutter frequency for all values of the roll angular velocity.

\section{CONCLUSIONS}

In this study, the aircraft is considered as an unrestrained wing carrying fuselage at its semi span and some arbitrarily placed stores. The effect of the roll maneuver, one of the most popular flight maneuvers, on flutter of the unrestrained wing is considered. To this end, the complete aeroelastic equations for an isotropic, unrestrained Goland wing under roll maneuver are formulated. The equations include effects of both maneuver induced and flow induced forces.

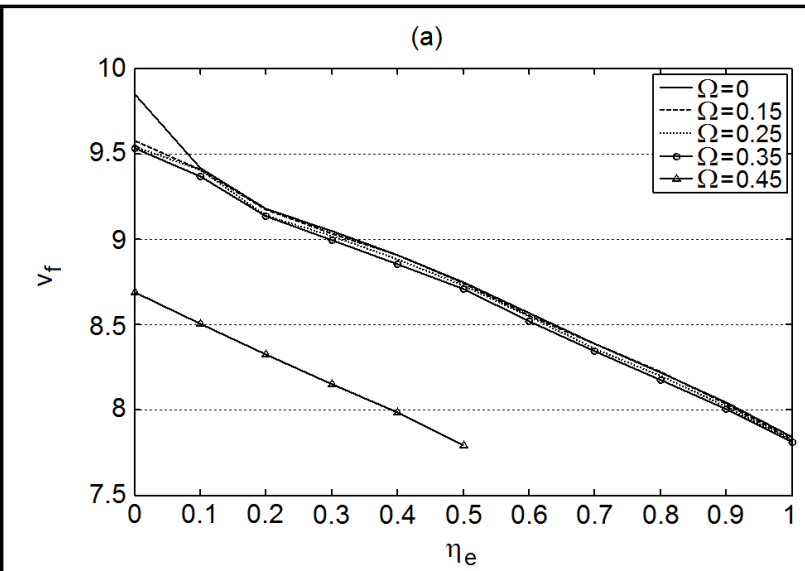

(b)

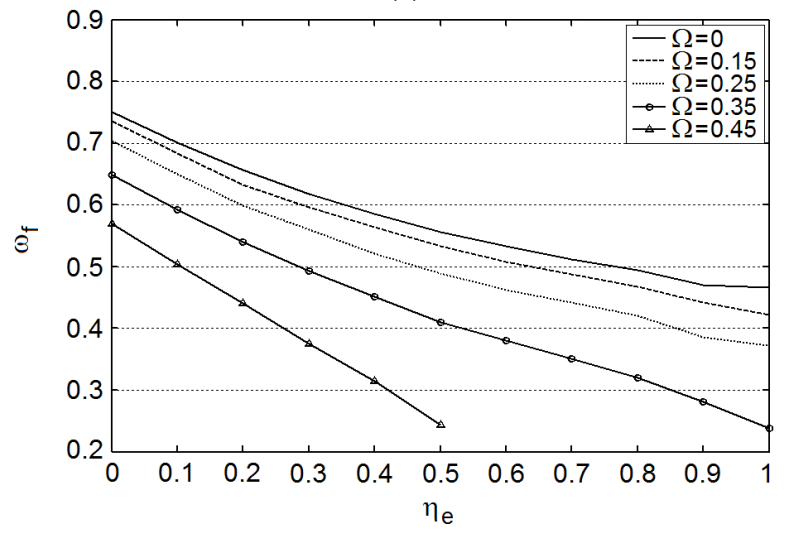

Figure 5. Effects of the store mass on the aircraft flutter boundary for $\eta_{F}=1$. (a) Flutter speed; (b) Flutter frequencies.

Results show the influence of the roll angular velocity, fuselage mass, and the stores mass and locations on the flutter speed of the wing. The rolling maneuver restricts the wing dynamic stability region in most of cases. Also, it is found that the flutter speed in the case of heavy stores is lower than those obtained for light ones, independent of the maneuver conditions.

\section{REFERENCES}

1 Goland, M. and Luke, Y. L. The flutter of a uniform wing with tip weights, Journal of Applied Mechanics, 15, 13-20, (1948).

2 Harry, L. R. and Charles, E. W. Flutter of a uniform wing with an arbitrarily placed mass according to a differential equation analysis and a comparison with experiment, NASA Technical Report, NACA TN-1848, (1949).

3 Lottati, T. A. Aeroelastic stability characteristics of a composite swept wing with tip weight for an unrestrained vehicle, Journal of Aircraft, 24 (11), 793-802, (1987). http://dx.doi.org/10.2514/3.45523

4 Gern, F. H. and Librescu, L. Static and dynamic aeroelasticity of advanced aircraft wings carrying external stores, AIAA Journal, 36, 1121-1129, (1998). http://dx.doi.org/10.2514/3.13944

5 Gern, H. and Librescu, L. Effect of externally mounted stores on aeroelasticity of advanced aircraft wings, 


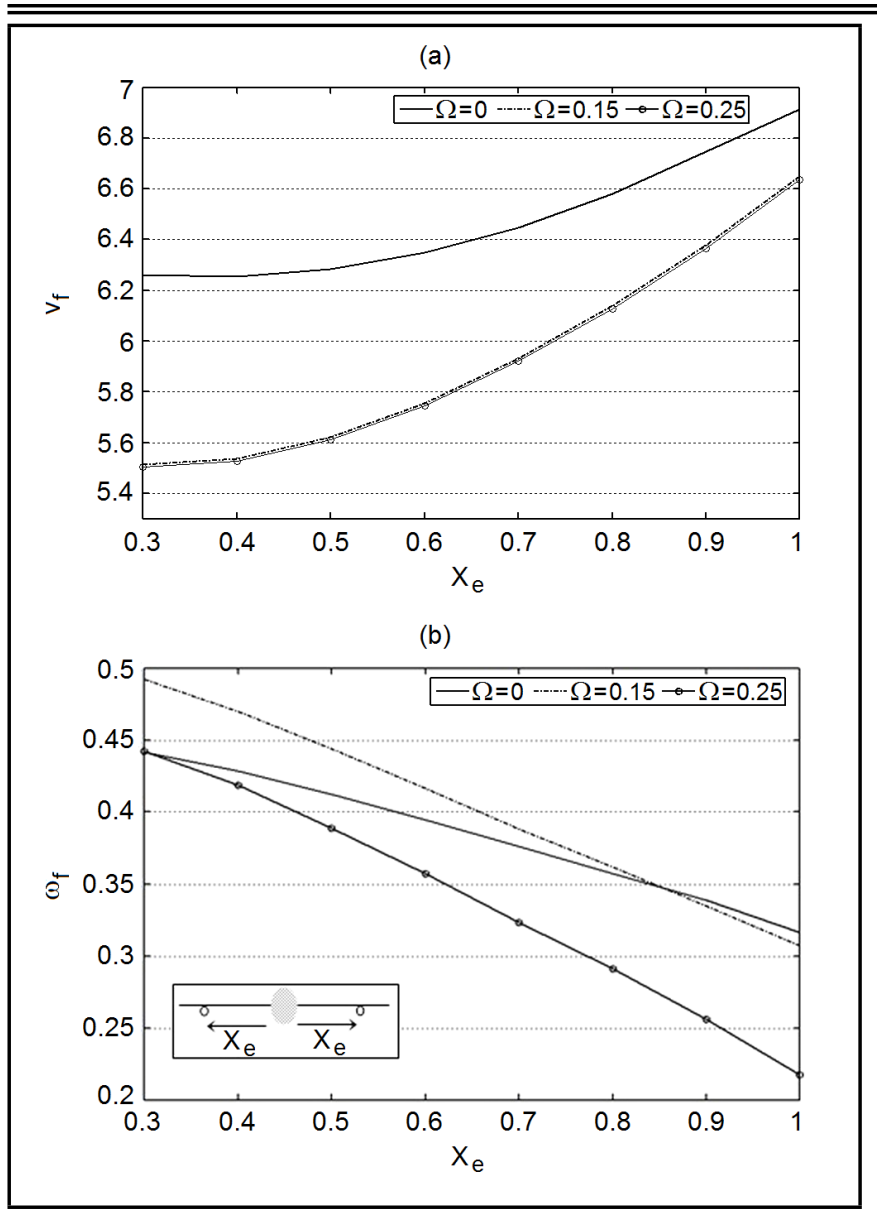

Figure 6. Effects of the spanwise position of the store on the aircraft flutter boundary for $\eta_{e}=\eta_{F}=1$. (a) Flutter speed; (b) Flutter frequencies.

Aerospace Science and Technology, 2 (5), 321-333, (1998). http://dx.doi.org/10.1016/s1270-9638(98)80008-4

$6 \mathrm{Na}$, S. and Librescu, L. Dynamic response of adaptive cantilevers carrying external stores and subjected to blast loading, Journal of Sound and Vibration, 231, 1039-1055, (2000). http://dx.doi.org/10.1006/jsvi.1999.2627

7 Librescu, L. and Song, O. Dynamics of composite aircraft wings carrying external stores, AIAA Journal, 46, 568-572, (2008). http://dx.doi.org/10.2514/1.25541

8 Edwards, J. W. and Wieseman, C. D. Flutter and divergence analysis using the generalized aeroelastic analysis method, Journal of Aircraft, 45 (3), 906-915, (2008). http://dx.doi.org/10.2514/1.30078

9 Fazelzadeh, S. A., Mazidi, A., and Kalantari, H. Bendingtorsional flutter of wings with an attached mass subjected to a follower force, Journal of Sound and Vibration, 323, 148162, (2009). http://dx.doi.org/10.1016/j.jsv.2009.01.002

10 Mazidi, A. and Fazelzadeh, S. A. The flutter of a swept aircraft wing with a powered-engine, Journal of Aerospace Engineering, 23, 243-250, (2010). http://dx.doi.org/10.1061/(asce)as.1943-5525.0000037

11 Fazelzadeh, S. A., Marzocca, P. A., Rashidi, E., and Mazidi, A. Effects of rolling maneuver on divergence and flutter of aircraft wing store, Journal of Aircraft, 47 (1), 64-70, (2010). http://dx.doi.org/10.2514/1.40463

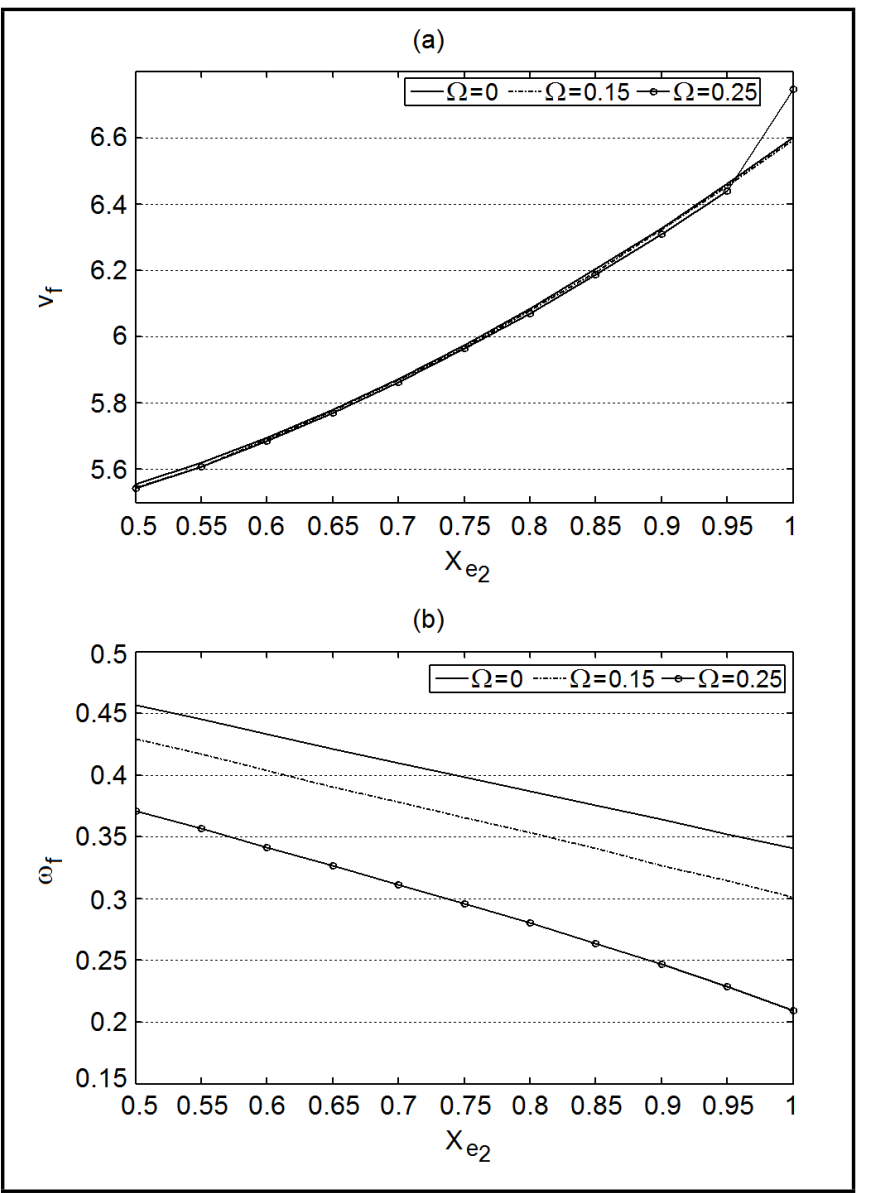

Figure 7. Effects of the spanwise position of the second engine on (a) the flutter speed and (b) the flutter frequency for $X_{e_{1}}=0.3$ and $\eta_{e_{1}}=\eta_{e_{2}}=$ $\eta_{F}=1$.

12 Mazidi, A., Fazelzadeh, S. A., and Marzocca, P. A. Flutter of aircraft wings carrying a powered engine under roll maneuver, Journal of Aircraft, 48, 874-884, (2011). http://dx.doi.org/10.2514/1.c031080

13 Baruh, H. Analytical Dynamics, McGraw-Hill, Boston, (1999).

14 Hodges, D. H. and Dowell, E. H. Nonlinear equations of motion for the elastic bending and torsion of twisted nonuniform rotor blades, NASA Technical Report, NASA TN D-7818, (1974).

15 Behera, R. K. and Parhi, R. K. Dynamic characteristics of a cantilever beam with transverse cracks, International Journal of Acoustics and Vibration, 11 (1), 3-18, (2006).

16 Hodges, D. H. and Pierce, G. A. Introduction to Structural Dynamics and Aeroelasticity, Cambridge University Press, Cambridge, (2002). http://dx.doi.org/10.1017/cbo9780511997112.002

17 Dowell, E. H., Crawley, E. F., Curtiss, H. C., Peters, D. A., Scanlan, R. H., and Sisto, F. A. Modern Course in Aeroelasticity, Kluwer Academic, Dordrecht, (1995).

18 Fletcher, C. A. J. Computational Galerkin Methods, Springer-Verlag, New York, (1984). http://dx.doi.org/10.1007/978-3-642-85949-6_2 